\title{
Virtual Microscopy using High-Resolution Image Datasets
}

\author{
Glenn Fried,* Benjamin Grosser, ${ }^{*}$ Tom Gaskins, ${ }^{* *}$ Alexandre Klementiev* \\ * Beckman Institute for Advanced Science and Technology \\ University of Illinois at Urbana-Champaign \\ 405 North Mathews, Urbana, IL 61801 \\ **3 15646 NE 100th Way, Redmond WA, 98052
}

Microscopes open the worlds of biology, nanotechnology, and the physical sciences to researchers, students, and the public. Unfortunately, instrumentation that produces the most useful and high-resolution images are also generally the most expensive, resulting in limited access. To some degree this can be overcome by collecting micrographs that capture the important features of a particular sample, but these individual images often lack detail or context. Functional virtual microscopes provide a low cost alternative to high-cost physical microscopes by providing the ability to examine samples at varying magnifications and focal planes.

Our first implementation is a Virtual Scanning Electron Microscope (VSEM). The VSEM provides the user with a virtual instrument that has the feel of a real Scanning Electron Microscope (SEM) by presenting a high-resolution SEM dataset that can be manipulated as if it was a sample in the SEM. This dataset is a collection of thousands of overlapping individual SEM images, automatically collected using custom software. At each location we also collect a series of images at several focus levels. These images are then tiled into one large image, lower magnifications are derived via interpolation, and finally cut back into small tiles to facilitate high-performance rendering. The dataset is stored in a simple folder hierarchy with an XML file defining its layout. The result is a single specimen file that can be accessed by the VSEM application.

The VSEM application contains controls for contrast, brightness, magnification, focus, and navigation - allowing the operator to move around the sample, zooming in on features of interest just as a user of a physical instrument would do. Since the image data is divided into small tiles, the VSEM application is only required to manipulate a small part of the data collected from a given sample at one time. This method of managing high-resolution images using small tiles chunked into and out of memory as needed allows the application to work on a typical desktop computer. The result is that users can easily and efficiently navigate though samples containing hundreds of megabytes of image data.

In addition to the VSEM we have also built a Virtual Light Microscope (VLM) and a Virtual Scanning Probe Microscope (VSPM) based on the same principles. Sample labeling tools have been incorporated into these virtual instruments to allow a specialist with knowledge of the sample's features to compose overlays that convey their knowledge to students. At the same time we envision students using the labeling tools 
themselves to answer questions about the samples in an educational setting. Instructional videos have been created for each virtual instrument covering the basics of how each microscope works, and a second video conveys the typical scientific applications for the microscope.

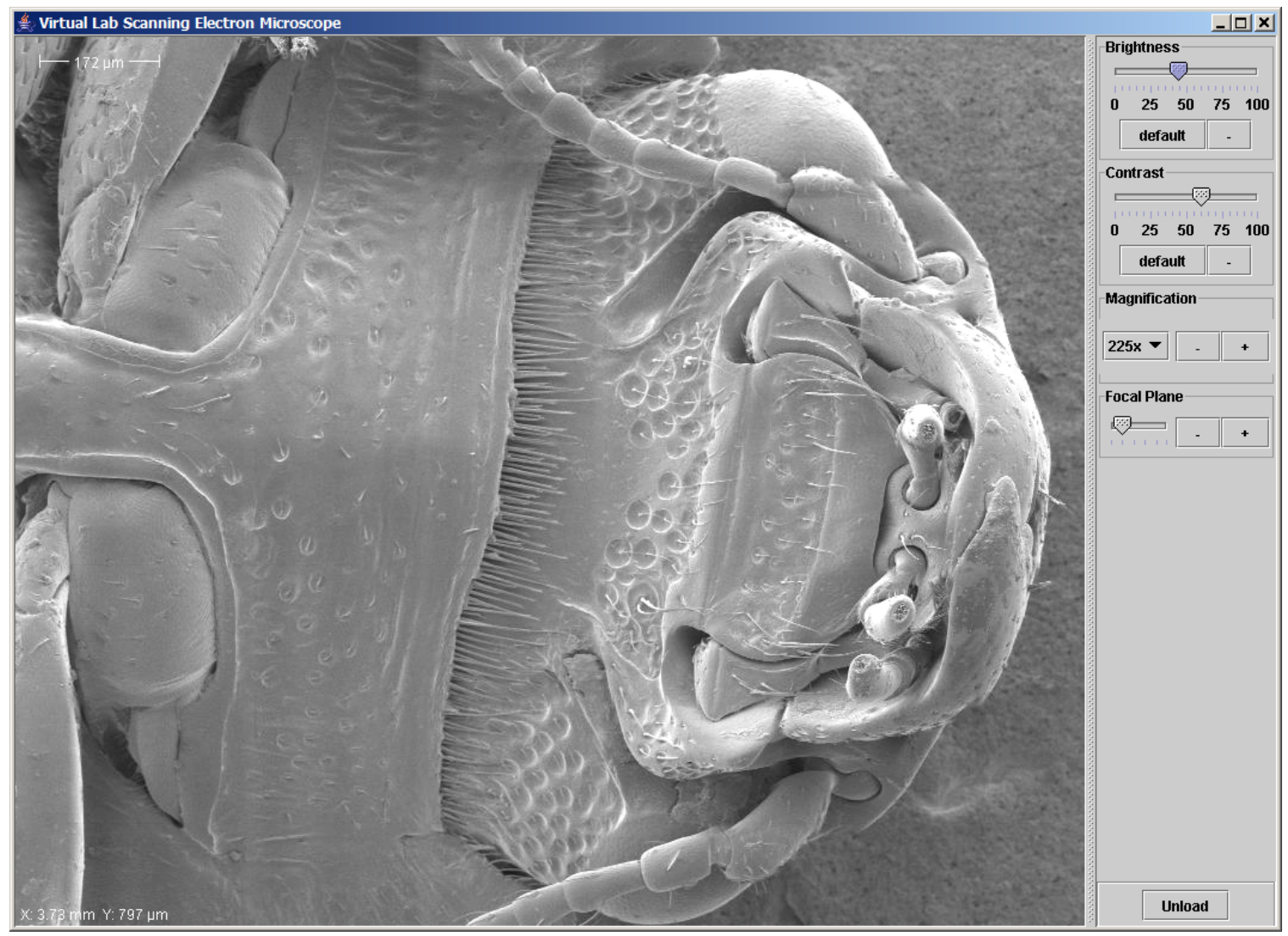

Figure 1: The Virtual Scanning Electron Microscope (VSEM) interface, with a beetle sample loaded. Navigation is controlled by clicking and holding the mouse in the desired direction. Focus, contrast, and brightness are controlled by the sliders, and magnification is controlled by the pull down menu or + and - buttons. The unload button takes the operator back to the sample selection screen. All of these controls are designed to be similar to a Philips XL 30 ESEM - the instrument used to collect the data. 